MIU-THP-94/68

June, 1994

\title{
Using Gauge Coupling Unification and Proton Decay to Test Minimal SuPERSYMMETRIC SU(5)
}

\author{
John S. Hagelin, S. Kelley \\ and Veronique Ziegler \\ Department of Physics \\ Maharishi International University \\ Fairfield, IA 52557-1069, USA
}

\begin{abstract}
We derive a one-loop expression, including all thresholds, for the mass of the proton decay mediating color triplets, $M_{D^{c}}$, in minimal supersymmetric $\mathrm{SU}(5)$. The result for $M_{D^{c}}$ does not depend on other heavy thresholds or extra representations with $\mathrm{SU}(5)$ invariant masses which might be added to the minimal model. We numerically correct our result to two-loop accuracy. Choosing inputs to maximize $M_{D^{c}}$ and $\tau_{P}$, within experimental limits on the inputs and a $1 \mathrm{TeV}$ naturalness bound, we derive a strict bound $\alpha_{3}>0.117$. We discuss how this bound will change as experimental limits improve. Measurements of $\alpha_{3}$ from deep inelastic scattering and the charmonium spectrum are below the bound $\alpha_{3}>0.117$ by more than $3 \sigma$. We briefly review several ideas of how to resolve the discrepancy between these low values of $\alpha_{3}$ and the determinations of $\alpha_{3}$ from LEP event shapes.
\end{abstract}

MIU-THP-94/68

June, 1994 
A common criticism of unified theories is that physics at a grand-unified scale of $10^{16} \mathrm{GeV}$ or a string-unified scale of $10^{18} \mathrm{GeV}$ cannot be tested. Indeed, a crucial ingredient of unified models is the accurate reproduction of the highly successful Standard Model predictions. However, precision measurements at low energies can probe physics at much higher energies. Proton decay [1], gauge coupling unification [2], flavor changing neutral currents [3], and sparticle spectroscopy [4] all provide windows to physics at unified scales which can be used to discriminate between different theories. The minimal non-supersymmetric GUT has already been ruled out by constraints from gauge coupling unification and proton decay. The purpose of this paper is to constrain the allowed parameter space of minimal supersymmetric SU(5) [5] from the combined experimental constraints on the lowenergy gauge couplings and proton decay. We obtain a very general result that $\alpha_{3}>0.117^{*}$ in the minimal model. This result also holds for extended models containing the minimal model field content plus extra representations with $\mathrm{SU}(5)$ invariant masses.

Although this limit is comfortably within the range of $\alpha_{3}$ extracted from LEP event shapes [6,7], measurements of $\alpha_{3}$ from deep inelastic scattering [8], and the charmonium spectrum [9] fall at least $3 \sigma$ below this bound on $\alpha_{3}$. This disagreement between different types of measurements of $\alpha_{3}$ has not been resolved, though several interesting proposals exist $[10,11,12,13]$. The situation is similar to that in the electroweak sector, where different types of measurements do not agree unless radiative corrections are properly included. However, radiative corrections to the strong coupling are more difficult. The definitive resolution of conflicting measurements of $\alpha_{3}$ is an extremely important project. If the real value of $\alpha_{3}$ lies close to that presently extracted from deep inelastic scattering and the charmonium spectrum, we will have ruled out yet another GUT. If the LEP event shape values are correct, supersymmetric SU(5) will survive even if proton decay is not observed in the next round of experiments.

Minimal supersymmetric $\mathrm{SU}(5)$ is an amazingly complete model which predicts the Standard Supersymmetric Model (SSM) as its low-energy limit. However, it is not a final model. The fine-tuning problem could possibly be solved at the expense

* Note that $\alpha_{e}, \sin ^{2} \theta$, and $\alpha_{3}$ are in the $\overline{M S}$ scheme renormalized at $m_{Z}$ throughout this paper, except where otherwise indicated. 
of complications arising in non-minimal models. A second question is how to incorporate quantum gravity into the model. General considerations of quantum gravitational effects introduce additional uncertainties into the calculations and blur predictions [14]. String theory could ultimately quantify the effects of quantum gravity and resharpen the predictions. However, there are still obstacles to deriving useful predictions from the string, as well as obtaining the scalar adjoint required to break SU(5) [15].

Considering that minimal supersymmetric $\mathrm{SU}(5)$ is most likely not the complete theory of nature, why should one attempt to seriously test it against experiment? We feel that the most important reason is to develop tools and techniques, which will be useful for more complete models, in the context of a simple, definite model. It is difficult to develop techniques for testing string theories when there are thousands to choose from, each with many parameters beyond the present calculational ability to specify. By taking a specific, definite model like supersymmetric $\mathrm{SU}(5)$, which can be rigorously compared to experiment, we can get some idea of what must be done to test string models.

In the step approximation for thresholds, the $S U(3) \times S U(2) \times U(1)$ couplings should unify at a scale $M_{X}=\max \left(M_{V}, M_{\Sigma}, M_{D^{c}}\right)$ where $V$ denotes the superheavy vector superfields, $\Sigma$ denotes the superheavy adjoint $\mathrm{SU}(3)$ and $\mathrm{SU}(2)$ remnants of the adjoint higgs, and $D^{c}$ denotes the proton-decay mediating color triplets. From this unification condition, the following predictions result [16].

$$
\begin{gathered}
\ln \left(\frac{M_{X}}{m_{Z}}\right)=\frac{\pi}{6}\left(\frac{3}{5 \alpha_{e}}-\frac{8}{5 \alpha_{3}}\right)+\sum_{l} C_{m}(l) \ln \left(\frac{m_{l}}{m_{Z}}\right)-\sum_{h} C_{m}(h) \ln \left(\frac{M_{X}}{M_{h}}\right)+\delta_{m} \\
\sin ^{2} \theta=0.2+\frac{7 \alpha_{e}}{15 \alpha_{3}}+\frac{\alpha_{e}}{20 \pi}\left[\sum_{l} C_{s}(l) \ln \left(\frac{m_{l}}{m_{Z}}\right)-\sum_{h} C_{s}(h) \ln \left(\frac{M_{X}}{M_{h}}\right)\right]+\delta_{s} \\
\frac{1}{\alpha_{X}}=\frac{1}{4}\left(\frac{3}{5 \alpha_{e}}+\frac{12}{5 \alpha_{3}}\right)+\frac{1}{20 \pi}\left[\sum_{l} C_{a}(l) \ln \left(\frac{m_{l}}{m_{Z}}\right)-\sum_{h} C_{a}(h) \ln \left(\frac{M_{X}}{M_{h}}\right)\right]+\delta_{a}
\end{gathered}
$$


where the sums over the light $m_{l} \gtrsim m_{Z}$, and heavy $M_{h} \lesssim M_{X}$, thresholds,

$$
\begin{aligned}
C_{m} & =\frac{b_{y}}{12}+\frac{b_{2}}{20}-\frac{2}{15} b_{3} \\
C_{s} & =\frac{10}{3} b_{y}-8 b_{2}+\frac{14}{3} b_{3} \\
C_{a} & =\frac{5}{2} b_{y}+\frac{3}{2} b_{2}+6 b_{3}
\end{aligned}
$$

and the correction terms $\delta_{i}=\delta_{i}($ gauge $)+\delta_{i}($ Yukawa $)$ include two-loop gauge and Yukawa effects. Choosing the $\delta_{i}$ (gauge) linear in $\alpha_{3}$ so that the analytic approximations, Equations (1.a,b,c), match numerical two-loop calculations at $\alpha_{3}=0.11$ and $\alpha_{3}=0.13$ gives

$$
\begin{aligned}
\delta_{m}(\text { gauge }) & =-0.0868852-1.1444784 \alpha_{3} \\
\delta_{s}(\text { gauge }) & =0.00127231+0.0147977 \alpha_{3} \\
\delta_{a}(\text { gauge }) & =-0.2385283-3.6119007 \alpha_{3}
\end{aligned}
$$

Figure 1 shows that this works extremely well for experimentally interesting values of $\alpha_{3}$.

Equations (1.a) and (1.b) can be used to solve for the mass of the proton-decay mediating triplet [17]:

$$
\begin{aligned}
\ln \left(\frac{M_{D^{c}}}{m_{Z}}\right) & =\frac{3 \pi}{\alpha_{e}}\left(0.2324 \pm 0.0003-1.03 \times 10^{7}\left[m_{t}^{2}-(138 G e V)^{2}\right]-\frac{5 \alpha_{e}}{9 \alpha_{3}}-\frac{1}{6}\right) \\
& +\sum_{l} C_{d}(l) \ln \left(\frac{m_{l}}{m_{z}}\right)-\sum_{h-D^{c}} C_{d}(h) \ln \left(\frac{M_{X}}{M_{h}}\right)+\delta_{d}
\end{aligned}
$$

where

$$
\begin{aligned}
C_{d} & =C_{m}-\frac{3}{20} C_{s}=\frac{-5}{12} b_{y}+\frac{5}{4} b_{2}-\frac{5}{6} b_{3} \\
\delta_{d} & =-\frac{3 \pi}{\alpha_{e}} \delta_{s}+\delta_{m}
\end{aligned}
$$

and we have used the experimental value of $\sin ^{2} \theta$ with explicit top mass dependence $[18]$. 
Notice that $C_{d}$ vanishes for the representation content of $M_{V}$ and $M_{\Sigma}$ [17]. This means that $M_{D^{c}}$ only depends on the low-energy parameters in the model and not on the other heavy scales. Additional representations beyond the minimal model with SU(5) invariant masses will not change this result. Therefore, in Eq. (4)

$$
\sum_{h-D^{c}} C_{d}(h) \ln \left(\frac{M_{X}}{M_{h}}\right)=0 .
$$

Writing out the contribution from light thresholds to Eq. (4) gives

$$
\sum_{l} C_{d}(l) \ln \left(\frac{m_{l}}{m_{Z}}\right)=\frac{11}{24} \ln \left(\frac{m_{t}}{m_{Z}}\right)+\frac{5}{3} \ln \left(\frac{m_{\tilde{w}}}{m_{\tilde{g}}}\right)+\frac{1}{6} \ln \left(\frac{m_{h}}{m_{Z}}\right)+\frac{2}{3} \ln \left(\frac{\mu}{m_{Z}}\right)+g_{d}(y, w)
$$

where $g_{d}(y, w)$ includes the threshold effects of squarks and sleptons [19].

To derive the most conservative bound, we must maximize Eq. (4) within the experimental limits of the inputs. Calculations of Yukawa effects on gauge coupling unification give [18]

$$
\delta_{s}(Y u k a w a)>-0.0004, \quad \delta_{m}(Y u k a w a)<0.06
$$

Taking into consideration experimental limits and using a $1 \mathrm{TeV}$ naturalness bound, $M_{D^{c}}$ is maximized by taking

$$
\begin{gathered}
\sin ^{2} \theta=.2327-1.03 \times 10^{-7}\left(m_{t}^{2}-138^{2} \mathrm{GeV}^{2}\right) \\
\alpha_{e}=\frac{1}{127.8} \\
m_{t}=130 \mathrm{GeV} \\
m_{h}=\mu=1 \mathrm{TeV} \\
g(y, w)=0^{*}
\end{gathered}
$$

*Because $C_{m}, C_{s}$, and $C_{d}$ vanish for complete $S U(5)$ representation, $g_{m}, g_{s}$, and $g_{d}$, which include the threshold corrections for the squarks and sleptons, are close to zero over the whole parameter space. Under simple assumptions for the stop mass matrix, $\max \left[g_{d}(y, w)\right]=0$. 
The threshold effects of the gauginos require more careful consideration. Since

$$
m_{\tilde{i}}=m_{1 / 2} \frac{\alpha_{i}\left(m_{\tilde{i}}\right)}{\alpha_{X}}
$$

we have

$$
\frac{m_{\tilde{w}}}{m_{\tilde{g}}}=\frac{\alpha_{2}\left(m_{\tilde{w}}\right)}{\alpha_{3}\left(m_{\tilde{g}}\right)}
$$

Below the wino threshold, $b_{2}$ is negative and $\alpha_{2}$ decreases. Therefore, $\alpha_{2}$ at the wino mass is bounded by

$$
\alpha_{2}\left(m_{\tilde{w}}\right)<\alpha_{2}\left(m_{Z}\right) .
$$

Below the gluino threshold, $b_{3}>-5$. Therefore $\alpha_{3}$ at a gluino mass less than $1 \mathrm{TeV}$ is bounded by

$$
\alpha_{3}\left(m_{\tilde{g}}\right)>\frac{\alpha_{3}\left(m_{Z}\right)}{1+\frac{5}{2 \pi} \alpha_{3}\left(m_{Z}\right) \ln \left(\frac{1 T e V}{m_{Z}}\right)} .
$$

This results in a bound

$$
\frac{m_{\tilde{w}}}{m_{\tilde{g}}}<\frac{\alpha_{e}}{\sin ^{2} \theta \alpha_{3}}\left[1+\frac{5}{2 \pi} \alpha_{3} \ln \left(\frac{1 \mathrm{TeV}}{m_{Z}}\right)\right]=\frac{0.0336}{\alpha_{3}}+0.0641
$$

Finally, the resulting bound on $M_{D^{c}}$ as a function of the strong coupling is

$$
\ln \left(\frac{M_{D^{c}}}{m_{Z}}\right)<80.8831-\frac{5 \pi}{3 \alpha_{3}}-18.9705 \alpha_{3}+\frac{5}{3} \ln \left[\frac{0.0336}{\alpha_{3}}+0.0641\right]
$$

Turning to proton decay, a detailed exploration of proton decay reveals that with a $1 \mathrm{TeV}$ naturalness bound, the most conservative bound on $M_{D^{c}}$ is given by $[17]$

$$
\ln \left(\frac{M_{D^{c}}}{m_{Z}}\right)>31.7
$$

This result uses a bound of $0.0003<\beta / \mathrm{GeV}^{3}<0.003$ on the hadronic matrix element. We will use a value of the hadronic matrix element from lattice calculations 
$\beta=(5.6 \pm 0.5) \times 10^{-3} \mathrm{GeV}^{3}[20]$ which gives a bound on $M_{D^{c}}$

$$
\ln \left(\frac{M_{D^{c}}}{m_{Z}}\right)>32.2
$$

Figure 2 plots the two bounds (15) and (17) in the $M_{D^{c}}, \alpha_{3}$ plane revealing that minimal supersymmetric SU(5) is compatible with proton decay and coupling constant only if $\alpha_{3}>0.117$. This analytic result matches well with $\alpha_{3}>0.118$ from a similar numerical calculation over large regions of the $\mathrm{SU}(5)$ parameter space [21].

One would not expect our analytic bound to be saturated in a full numerical calculation for any point in $\mathrm{SU}(5)$ parameter space. This is because our result uses an upper bound on $M_{D^{c}}$ from coupling constant unification and a lower bound on $M_{D^{c}}$ from proton decay which are independent, and each bound has been extremized for each input separately. Because of correlations in the spectrum, neither bound is likely to be saturated nor are the upper and lower bounds likely to be simultaneously realized. Our analytic bound is thus very conservative and can be trusted over the whole parameter space of the model without questioning the intricacies of a computerized search over a large dimension parameter space.

It is interesting to see the effect of improvements in the various experimental bounds on the minimum value of $\alpha_{3}$ compatible with minimal supersymmetric $\mathrm{SU}(5)$. A factor of ten improvement in the bounds on the proton lifetime would give $\alpha_{3}>0.120$, while a top quark mass of $m_{t} \geq 175 \mathrm{GeV}$ would give $\alpha_{3}>0.121$. Both a tenfold improvement in the proton lifetime bound and a top mass $m_{t} \geq 175 \mathrm{GeV}$ would give $\alpha_{3}>0.125$. Since the bound on $M_{D^{c}}$ from proton decay is proportional to $m_{\tilde{w}} / m_{\tilde{q}, \tilde{l}}^{2}$, an increase in the upper bound on the wino mass or evidence that the squarks and sleptons are below $1 \mathrm{TeV}$ would result in a more stringent bound on $\alpha_{3}$. A factor of four increase in $m_{\tilde{w}} / m_{\tilde{q}, \tilde{l}}^{2}$ would give $\alpha_{3}>0.121$. Conversely a factor of four decrease in $m_{\tilde{w}} / m_{\tilde{q}, \tilde{l}}^{2}$ due to an increase in the naturalness bound from $1 \mathrm{TeV}$ to $2 \mathrm{TeV}$ would give $\alpha_{3}>0.113$.

From an experimental standpoint, even the bound $\alpha_{3}>0.125$ is compatible with present extractions of $\alpha_{3}$ from LEP event shapes. Values of $\alpha_{3}=0.120 \pm 0.006$ from OPAL [6] and $\alpha_{3}=0.123 \pm 0.006$ from DELPHI [7] represent typical central values and error bars, although the central values are sensitive to what observable 
is used and the details of the analysis method. However, there are indications that the strong coupling might be much smaller. Analysis of deep ineleasic scattering data gives $\alpha_{3}=0.108 \pm 0.002$ [8]. Lattice gauge theory calculations based on the 1P-1S splitting in charmonium give $\alpha_{3}=0.105 \pm 0.004$ [9]. Resolving the discrepency between the high energy measurements of $\alpha_{3}$ at LEP and various low energy measurements has been an important issue in GUTS for some time [22].

Tree level values of couplings from different experiments should not match precisely; radiative corrections must be included. The precision of different measurements of electroweak parameters has reached the sensitivity where theory is being rigourosly tested at the loop level, and radiative corrections must be properly included to see the consistancy of different types of measurements [23]. It is likely that there is a similar, though more computationally intensive, explanation of the discrepencies between different measurements of $\alpha_{3}$. Several ideas regarding the details of this explanation already exist. A proposed method for summing the higher-order corrections to LEP event shapes reduce the LEP event shape extractions of $\alpha_{3}$ by about 0.012 [10]. Attempts to systematically resolve the scale and scheme ambiguities [11] in determining $\alpha_{3}$ have resulted in a value of $\alpha_{3}=0.107 \pm 0.003$ extracted from LEP event shapes [12]. A primary motivation for the light gluino is the reconciliation of different measurements of $\alpha_{3}$ due to the modification of the running of $\alpha_{3}$ in the presence of light gluinos [13]. With light gluinos, deep inelastic scattering measurements give $\alpha_{3}=0.124 \pm 0.001$ [8].

However, in the context of radiative electroweak breaking in the SSM, which minimal supersymmetris $\mathrm{SU}(5)$ reduces to after integrating out the superheavies [24], the light gluino scenario is in trouble! Simple considerations of the chargino and neutralino sector severely restrict the parameter space [25]. Moreover, explicit calculations of radiative electroweak breaking show that the small area of parameter space compatible with other experimental limits has a top mass of about $114 \mathrm{GeV}$ [26], which is well below present experimental limits [27]. Nonuniversal supersymmetry breaking may be able to save the light gluino scenario. However, because of the large ratio of $m_{\tilde{q}} / m_{\tilde{g}}$, the light gluino scenario gives a relic density hundreds of times larger than compatible with the age of the universe. Introducing $\mathrm{R}$ parity breaking into the model would probably be required to remedy this dark matter problem. 
In conclusion, we have deduced a conservative bound, $\alpha_{3}>0.117$, in the minimal supersymmetric SU(5) model. Confirmation of a high top mass and improvements in proton lifetime bounds would substantially increase this bound. Although compatible with values of $\alpha_{3}$ extracted from LEP event shapes, the bound is in contradiction with measurements of $\alpha_{3}$ from deep inelastic scattering [8] and the charmonium spectrum [9]. Resolution of the discrepancies between different measurements of the strong coupling is of extreme importance for testing GUT scale physics. Although proper incorporation of radiative corrections to the measurements of $\alpha_{3}$ will most likely remove all discrepancies, there is no concensus on the details. The light gluino scenario brings most measurements of $\alpha_{3}$ into agreement with those from LEP event shapes. However, the light gluino scenario has severe phenomenological problems.

Unless the gluino is light or another source of radiative correction substantially increases the value of $\alpha_{3}$ extrated from deep inelastic scattering and the charmonium spectrum, the bound $\alpha_{3}>0.117$ may signal the demise of minimal supersymmetric SU(5).

Acknowledgements: We thank H. Pois and B. Wright for useful discussions. The work of John Hagelin is supported in part by National Science Foundation under grant No. PHY-9118320. 


\section{REFERENCES}

[1] H. Georgi and S.L. Glashow, Phys. Rev. Lett. 32(1974) 438.

[2] H. Georgi, H. Quinn and S. Weinberg, Phys. Rev. Lett. 33 (1974) 451.

[3] H. Georgi, Phys. Lett. 169B (1986) 231;

L.J.Hall, V.A. Kostelechy and S. Raby, Nucl. Phys. B 267 (1986) 415.

[4] A.E. Faraggi, J.S. Hagelin, S. Kelley and D.V. Nanopoulos, Phys. Rev. D 45 (1991) 3272.

[5] S. Dimopoulos and H. Georgi, Nucl. Phys. B 193 (1981) 150;

N. Sakai, Z. Phys. C 11 (1982) 153;

A. Chamseddine, R. Arnowitt and P.Nath, Phys. Rev. Lett. 105 (1982) 150 .

[6] OPAL collaboration, Z. Phys. C 59 (1993) 1.

[7] DELPHI collaboration, Z. Phys. C 59 (1993) 21.

[8] J. Blumlein, J. Botts, Phys. Lett. B 325 (1994) 190.

[9] A.X. Khadra, G. Hockney, A.S. Kronfeld and P.B. Mackenzie, Phys. Rev. Lett. 69 (1992) 729.

[10] J. Ellis, D.V. Nanopoulos and D.A. Ross, Phys. Lett. B267 (1991) 132.

[11] S.J. Brodsky and H.J. Lu, Preprint SLAC-PUB 6389 (1993).

[12] G. Kramer and B. Lampe, Z. Phys. 339 (1991) 189.

[13] J. Ellis, D.V. Nanopoulos and D.A. Ross, Phys. Lett. B 305 (1993) 375.

[14] L.J. Hall and U. Sarid, Phys. Rev. Lett. 70 (1993) 2673.

[15] H. Dreinel, J.L. Lopez, D.V. Nanopoulos and D. Reisa, Phys. Lett. B216 (1989) 289.

[16] J. Ellis, D.V. Nanopoulos and S. Kelley, Phys. Lett. B 260 (1991) 131.

[17] J. Hisano, H. Murayama and J. Yanagida, Nucl.Phys. B 402 (1993) 46.

[18] P. Langacker and N. Polonsky, Phys. Rev. D 47 (1993) 4028. 
[19] J. Ellis, D.V. Nanopoulos and S. Kelley, Nucl. Phys. B 373 (1992) 55.

[20] M.B. Gavela et al., Nucl Phys. B312 (1989) 269.

[21] B. Wright, Preprint MAD/PH/812 (1994).

[22] J.S. Hagelin and S. Kelley, Preprint MIU-THP-92/61 (1992).

[23] G. Altarelli, Preprint CERN-TH.7072/93 (1993).

[24] J.L. Lopez, D.V. Nanopoulos, H. Pois, S. Kelley and K. Yuang, Nucl. Phys. B 98 (1993) 3.

[25] L. Clavelli, P. Coutter and K. Yuang, Phys. Rev. D 47 (1993) 1973.

[26] J.L. Lopez, D.V. Nanopoulos and X. Wang, Phys. Lett. 313 (1993) 241.

[27] CDF Collaboration, Preprint FERMILAB-PUB-94-097-E (1994).

\section{Figure Captions}

(1) Percentage differences between the fitted analytic results and the explicit numerical calculations for $\sin ^{2} \theta, \ln \left(\frac{M_{X}}{m_{Z}}\right)$ and $\alpha_{X}$. The fit is chosen so that the difference vanishes at $\alpha_{3}=0.11$ and $\alpha_{3}=0.13$.

(2) The allowed region in the $M_{D^{c}}, \alpha_{3}$ plane for the minimal supersymmetric $\mathrm{SU}(5)$ model. The upper bound on $M_{D^{c}}$ is from gauge coupling unification, and the lower bound on $M_{D^{c}}$ is from proton decay. The minimal supersymmetric $\mathrm{SU}(5)$ model is only compatible with values of $\alpha_{3}$ greater than 0.117 . 